\title{
Effects of the vascular endothelial growth factor receptor-2 (VEGFR-2) inhibitor SU5416 on in vitro cultures of Plasmodium falciparum
}

\author{
Casper Hempel ${ }^{1,2+}$, Nils Hoyer ${ }^{1,2^{*}+}$, Trine Staals $\varnothing^{1,2}$ and Jørgen A Kurtzhals $s^{1,2}$
}

\begin{abstract}
Background: Vascular endothelial growth factor (VEGF) is taken up by parasitized red blood cells during malaria and stimulates intra-erythrocytic growth of Plasmodium falciparum in vitro. The cause and consequence of this uptake is not understood.
\end{abstract}

Methods: Plasmodium falciparum was cultured in vitro. Parasite growth and intracellular VEGF levels were assessed using flow cytometry. Intracellular VEGF was visualized by fluorescence immunocytochemistry. Phosphorylated tyrosine was measured by western blotting. In vivo assessment of intra-erythrocytic VEGF was performed in Plasmodium berghei ANKA-infected C57BL/6 mice.

Results: VEGF accumulated intracellularly in infected red blood cells, particularly in schizonts. In vitro growth of P. falciparum was unchanged when co-cultured with the anti-VEGF antibody bevacizumab or with an anti-VEGF receptor-1 peptide. In contrast, the VEGF receptor-2 inhibitor, SU5416, dose-dependently inhibited growth. None of the treatments reduced intracellular VEGF levels. Thus, the anti-parasitic effect of SU5416 seemed independent of VEGF uptake. SU5416 reduced phosphorylated tyrosine in parasitized red blood cells. Similarly, the broad-spectrum tyrosine kinase inhibitor genistein dose-dependently inhibited P. falciparum growth and reduced tyrosine phosphorylation. Neither bevacizumab nor anti-VEGF receptor-1 peptide affected tyrosine kinase activity. Finally, in vivo uptake of VEGF in $P$. berghei ANKA was demonstrated, analogous to the in vitro uptake in $P$. falciparum, making it a possible model for the effects of VEGF signalling in vivo during malaria.

Conclusions: Inhibition of VEGFR-2 signalling reduces intra-erythrocytic growth of $P$. falciparum, likely due to tyrosine kinase inhibition. Internalisation of VEGF in $P$. falciparum-infected red blood cells does not rely on VEGF receptors. The function of in vivo uptake of VEGF can be studied in rodent malaria models.

Keywords: Plasmodium falciparum, Vascular endothelial growth factor, VEGF, Vascular endothelial growth factor receptor, Tyrosine kinase inhibitor, Plasmodium berghei ANKA

\section{Background}

Plasmodium falciparum malaria is responsible for over one million deaths annually, caused by complications like severe anaemia and cerebral malaria (CM). The clinical outcome of malaria is influenced by host genetics and parasite characteristics [1-3].

\footnotetext{
* Correspondence: nils.hoyer@gmail.com

${ }^{\dagger}$ Equal contributors

${ }^{1}$ Centre for Medical Parasitology, Department of Clinical Microbiology, Copenhagen University Hospital, Copenhagen, Denmark

2Department of International Health, Immunology and Microbiology, University of Copenhagen, Copenhagen, Denmark
}

Sequestration of parasitized red blood cells (PRBCs) in cerebral blood vessels, resulting in local hypoxia and neuronal damage, is a key event in the pathogenesis of $\mathrm{CM}$ [2]. The angiogenic and neuroprotective glycoprotein vascular endothelial growth factor (VEGF) can potentially be induced by these mechanisms. Indeed, it has been shown to be associated to malaria. In nonimmune travellers and Kenyan children with malaria, VEGF is increased in both brain tissue and blood [4,5]. Its release has mainly been linked to hypoxia [6] since its expression is stimulated via stabilization of hypoxia inducible factor (HIF)-1 $\alpha$ [7]. Also inflammation results in increased VEGF 
expression [8], and it may be a non-specific response to severe disease [9]. In human CM, histopathological analyses post mortem as well as studies on cerebral blood flow in comatose patients strongly support localized cerebral hypoxia, hypoperfusion, or both $[9,10]$. HIF-1 $\alpha$, which has a short half-life, was undetectable in human brain tissue post mortem, but a HIF- $1 \alpha$ associated protein, DEC-1 was upregulated in neurons [9]. Thus, sequestration of PRBCs followed by local cerebral hypoxia and accumulation of HIF- $1 \alpha$ is a possible cause of VEGF production during $\mathrm{CM}$.

There is an unexplained uptake of VEGF in a large fraction of PRBCs during both uncomplicated and severe malaria [9]. In fatal CM cases, the proportion of VEGFpositive PRBCs is proportional to the degree of parasite sequestration in cerebral blood vessels [9]. Addition of VEGF to in vitro cultured $P$. falciparum increases parasitaemia, implying that VEGF may be a trophic factor for the parasites [11]. VEGF uptake has been proposed to depend on VEGF-receptor-2 (VEGFR-2), since this receptor has been demonstrated on the red blood cell surface in serum-enriched cultures of $P$. falciparum [11]. Cultivation in serum-free medium reduced the trophic effect of VEGF concomitantly with a reduction of VEGFR2 on red blood cells [11]. VEGFR-2, a tyrosine kinase, is the major mediator of the antiapoptotic, mitogenic, angiogenic, and permeability-enhancing effects of VEGF on endothelial cells in adult persons and the expression of VEGFR-2 is increased during hypoxia [7].

This study was performed to investigate, whether direct inhibition of VEGF by the monoclonal anti-VEGF antibody bevacizumab or inhibition of VEGF receptors would reduce $P$. falciparum growth and prevent uptake of VEGF into PRBCs. Furthermore the in vivo uptake of VEGF was tested in the rodent malaria strain Plasmodium berghei ANKA, which serves as a mouse model of CM.

\section{Methods}

In vitro culture of Plasmodium falciparum

Plasmodium falciparum strain 3D7 was cultured in human serum-enriched medium according to standard methods [12]. Briefly, the parasites were grown in culture flasks at $37^{\circ} \mathrm{C}$ at $4 \%$ haematocrit in HEPES-buffered RPMI 1640 medium (Gibco, Life Technologies, Paisley, UK) supplemented with $10 \%$ human serum (blood group O), $0.05 \mathrm{mg} / \mathrm{ml}$ gentamycin (Gibco), $0.18 \mathrm{mg} / \mathrm{ml} \mathrm{L}$-glutamine (Sigma-Aldrich) in an atmosphere of $5 \% \mathrm{O}_{2}, 5 \% \mathrm{CO}_{2}$, and $90 \% \mathrm{~N}_{2}$. Throughout the study, parasites were subcultured by adding fresh group $\mathrm{O}$ red blood cells whenever parasitaemia reached $5 \%$. Human blood was drawn from healthy volunteers after obtaining verbal informed consent. Under Danish regulations, this did not require approval from an ethics committee. To produce serum, blood was allowed to clot. After centrifugation serum was aspirated, immediately frozen, and stored at $-20^{\circ} \mathrm{C}$ until used. All experiments were performed in triplicate and repeated at least three times, unless stated otherwise.

\section{Inhibition of VEGF, VEGFR-1 and VEGFR-2}

At day $0,50 \mu \mathrm{L}$ of a healthy malaria culture with a haematocrit of $50 \%$ and a parasitaemia of $0.4 \%$ was added to $150 \mu \mathrm{L}$ of culture medium in microtitre plates. Prior to seeding, PRBCs were enriched for ring stages by centrifugation on $5 \%$ sorbitol (Sigma-Adrich) as previously described [13]. Culture medium was carefully sampled and replaced by pre-warmed medium.

For direct VEGF inhibition, the humanized monoclonal anti-VEGF antibody bevacizumab (Avastin, Roche, Denmark) was added daily to the growth medium, resulting in the following concentrations in four different groups: $10 \mathrm{nM}, 100 \mathrm{nM}, 1,000 \mathrm{nM}$, and $10 \mu \mathrm{M}$. To allow for binding between bevacizumab and any VEGF in the growth medium, bevacizumab and growth medium were mixed at least one hour prior to addition to the culture. Growth medium with phosphate buffered saline (PBS) added instead of bevacizumab was used as control.

For VEGFR-1 inhibition, the anti-VEGFR-1 peptide which blocks the VEGF binding site on VEGFR-1 [14] (Anaspec, CA, USA) was added daily to the growth medium resulting in concentrations of $7 \mu \mathrm{M}, 22 \mu \mathrm{M}$, $67 \mu \mathrm{M}$, and $200 \mu \mathrm{M}$ respectively, at least one hour prior to addition to the culture. Growth medium with PBS added instead of anti-VEGFR-1 was used as control.

For VEGFR-2 inhibition, the VEGFR-2 inhibitor SU5416 (Tocris Bioscience, UK) was added daily to the growth medium, resulting in concentrations of $4 \mu \mathrm{M}, 20 \mu \mathrm{M}$, $100 \mu \mathrm{M}$, and $500 \mu \mathrm{M}$ respectively, at least one hour prior to addition to the culture. Growth medium with dimethyl sulphoxide (DMSO, Sigma-Aldrich, Denmark) added instead of SU5416 was used as control.

\section{Inhibition of tyrosine kinase activity}

For inhibition of tyrosine kinase activity a broad-acting inhibitor, genistein (Sigma-Aldrich, Denmark) was used. Genistein was used at four concentrations: 1, 4, 16, and $64 \mu \mathrm{g} / \mathrm{ml}$, which were prepared at least one hour prior to addition to the culture. Growth medium with DMSO added instead of genistein was used as control. These concentrations were also applied for growth inhibition assays.

\section{Immunocytochemistry}

Thin blood smears of cultured blood were fixed in a mixture of $25 \%$ acetone (Merck Chemicals, Germany) and 75\% absolute ethanol (Bie \& Berntsen A/S, Denmark). After fixation, slides were permeabilized with $0.1 \%$ triton x-100 (Sigma-Aldrich, Denmark) and blocked with 5\% bovine serum albumin (Sigma-Aldrich, Denmark) for one 
hour. Incubation for one hour with primary antibody (rabbit anti-VEGF Ab-1, ThermoScientific, Denmark) at a concentration of $0.01 \mathrm{mg} / \mathrm{mL}$, and secondary antibody (goat anti-rabbit Alexa Fluor 488, Life Technologies, Denmark) at a concentration of $0.5 \mu \mathrm{g} / \mathrm{mL}$, were followed by a nuclear stain (DAPI, Life Technologies). Samples without primary antibody served as negative controls. The negative control was further verified by pre-incubation where an excess (1.5x) of mouse VEGF (R\&D Systems, UK) was added to the primary antibody 0.5 hours prior to incubating on the thin blood smears. Slides were covered with anti fade reagent (Slow Fade Gold anti fade reagent, Life Technologies) and cover slipped.

\section{Fluorescence microscopy}

Light immunofluorescence microscopy photos were taken with an Olympus BX40 microscope equipped with an Olympus DP71 digital camera. DAPI (Olympus filter cube WU, excitation 330-385, emission 420) and fluorescein (3540B, excitation 482/35, emission 536/40, Olympus) filters were used. VIS (version 2.14.10.0) was used to acquire and save images. ImageJ (version 1.44i [15]) was used for further processing. Parasite development stage was determined by the number of parasite nuclei per red blood cell. Red blood cells with three or more distinguishable parasite nuclei were considered late stage.

\section{Confocal microscopy}

Confocal immunofluorescence microscopy photos were taken with a Nikon TE 2000E Eclipse, with 360 numerical aperture, 1.4 Apoplan oil immersion objective lens, with gain adjusted for each laser (515/30, 605/75). Z-stacks were generated with Nikon EZ-C1 software with z-steps of $0.15 \mu \mathrm{m}$.

\section{Flow cytometry}

Parasitaemia was determined daily by staining of samples with acridine orange (Sigma-Aldrich, Denmark) followed by flow cytometry as previously described [16]. Samples were processed on a FACSCanto flow cytometer (BD Biosciences, USA). Ten thousand events were recorded for each sample and the resulting scatter plots were analysed using the Weasel software (The Walter and Eliza Hall Institute of Medical Research, version 3.0).

For the detection of intracellular VEGF in PRBCs by flow cytometry at day 3 , when late-stage parasites were predominant, cells were fixed in $1 \%$ paraformaldehyde (Ampliqon, Denmark) for $10 \mathrm{~min}$ at room temperature. After washing in PBS, the cells were permeabilized in $0.1 \%$ saponin (Sigma-Aldrich, Denmark) for $10 \mathrm{~min}$. Intracellular VEGF was detected by incubating $5 \times 10^{5}$ cells for 40 min with mouse anti-human VEGF conjugated to allophycocyanin $(2 \mu \mathrm{g} / \mathrm{ml}$, clone $23410, \mathrm{R} \& \mathrm{D}$ Systems) according to manufacturer's instructions. The reactivity was compared with a relevant isotype control $(2 \mu \mathrm{g} / \mathrm{ml}$, $R \& D$ Systems). Fifty thousand events were recorded using the $633 \mathrm{HeNe}$ laser. The DNA binding dye Syto-9 (5 $\mu \mathrm{M}$, Life Technologies) was added to the staining solution. This made it possible to discriminate PRBCs, which contain DNA, from nonparasitized red blood cells, which do not contain DNA. The precision of this detection of PRBCs was comparable with acridine orange ( $p>0.61$ ). The $488 \mathrm{~nm}$ Argon laser was used for Syto-9. Data analyses were carried out using FlowJo version 7.6.5 (Three Star Inc, OR, USA).

\section{Western blotting}

At day 1, late-stage PRBCs were lysed in PhosphoSafe (Merck Chemicals, Germany) to preserve the current phosphorylation of proteins according to the manufacturer's instructions. The lysates were stored at $-20^{\circ} \mathrm{C}$ until western blotting was performed. Protein content was determined using the Lowry assay (DC protein assay, Bio-Rad, CA, USA). The proteins were separated on $10 \%$ polyacrylamide gels (BisTris mini, Life Technologies) at $150 \mathrm{~V}$ using MES buffer (Life Technologies). Twenty $\mu \mathrm{g}$ protein was loaded into each well and electrophoresis was carried out under denaturing and reducing conditions. Proteins were transferred to polyvinylidene membranes (ImmunBlot, Bio-Rad) at $30 \mathrm{~V}$ for one hour. The membranes were blocked with $5 \%$ bovine serum albumin (Sigma-Aldrich, Denmark) for one hour at room temperature before being incubated with an anti-phosphorylated tyrosine $(\mathrm{pTyr})$ antibody at $4^{\circ} \mathrm{C}$ overnight $(2000 \times$ dilution, Cell Signaling Technology, MA, USA). The primary antibody was detected using a goat anti-mouse horseradish peroxidase-conjugated secondary antibody (Dako, Glostrup, Denmark). Bands were detected using SuperSignal West Femto Substrate (Pierce, Thermo Fischer, IL, USA). The bands were quantified using open-access Fiji software [17]. The bands were normalized to total protein content of the well by shortly staining the membrane in $0.25 \%$ Coomassie Blue and destaining overnight according to standard methods before scanning the membrane in epi-illumination mode (Chemidoc XRS, Bio-Rad). This experiment was performed three times in duplicate.

\section{Animal experiments}

Female, pathogen-free, six- to eight-week old C57/Bl6 mice (Taconic, Ejby, Denmark) were infected by intraperitoneal (ip) injection of P. berghei ANKA (inoculum size $=10^{4}$ PRBCs). Animals were treated according to standard protocols and given food and water ad libitum. The experiment was approved by the Danish Animals Inspectorate (license number 2006/561-1128). On day 8 , peripheral blood was collected for thin blood smears and processed for immunocytochemistry as described. 

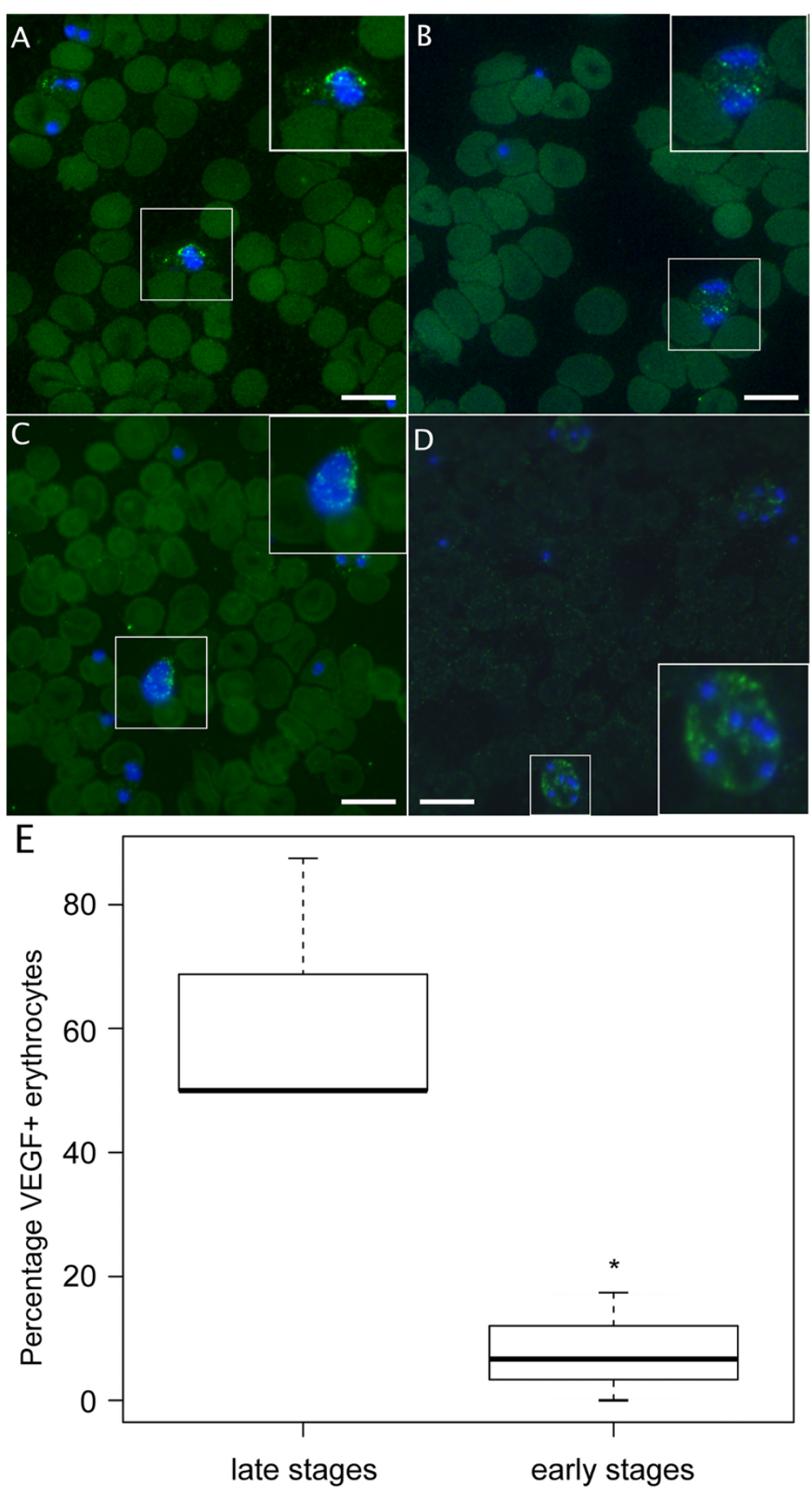

Figure 1 Immunocytochemistry (ICC) of cultured human red blood cells infected with Plasmodium falciparum and peripheral blood smears from mice infected with Plasmodium berghei ANKA show uptake of VEGF into late stage parasites, not inhibited by blocking VEGFR-2 or VEGF. Fluorescence microscopy of thin blood smears after culture of human blood infected with $P$. falciparum with no inhibition (A), SU5416 (B), or bevacizumab (C). DNA is stained blue by DAPI, VEGF is stained green. Uninfected red blood cells and most early stage parasitized red blood cells (containing a maximum of two DNA fragments) did not stain positive for VEGF. Inlets present $1.5 \times$ enlargement of marked area on the slide. Bar size $=10 \mu \mathrm{m}$. Thin blood smears taken from mice infected with P. berghei ANKA (D) showed similar staining compared to culture of human blood. Inlet presents $2 x$ enlargement of marked area on the slide. Bar size $=10 \mu \mathrm{m}$. Manual counting of parasitized red blood cells showed a significant increase in VEGF uptake in late stage PRBCs (containing more than two DNA fragments) compared to early stages $(p=0.038, \mathbf{E})$. 

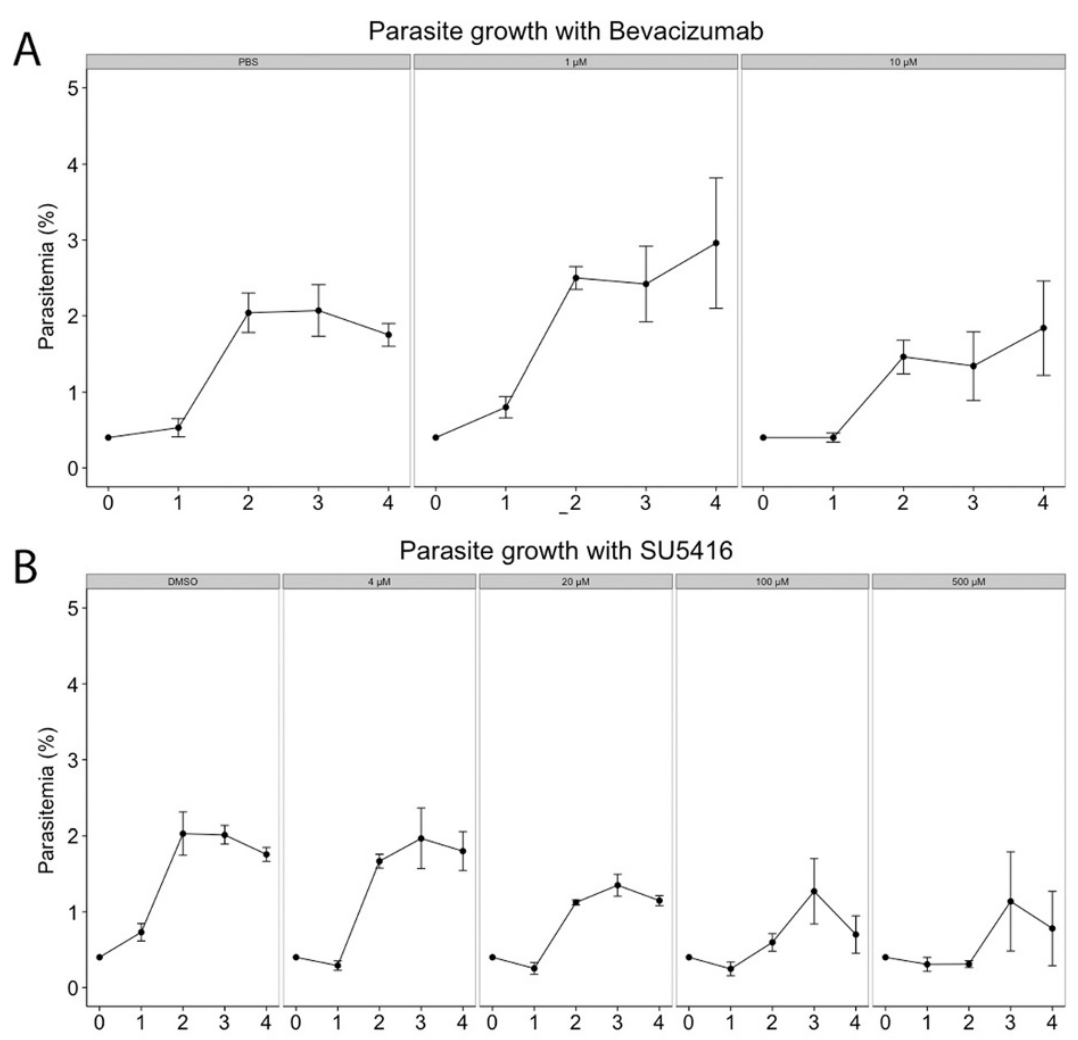

C
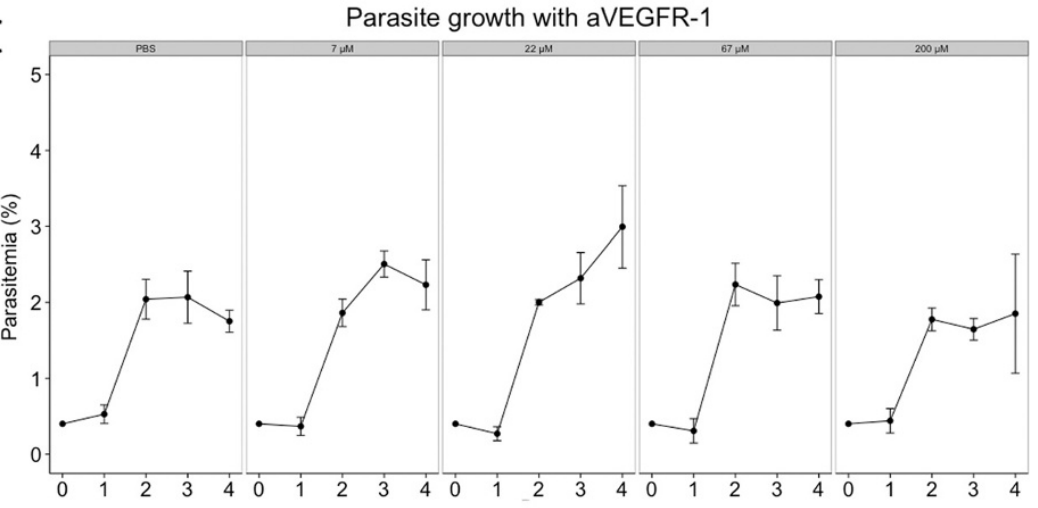

D Parasite growth with Genistein

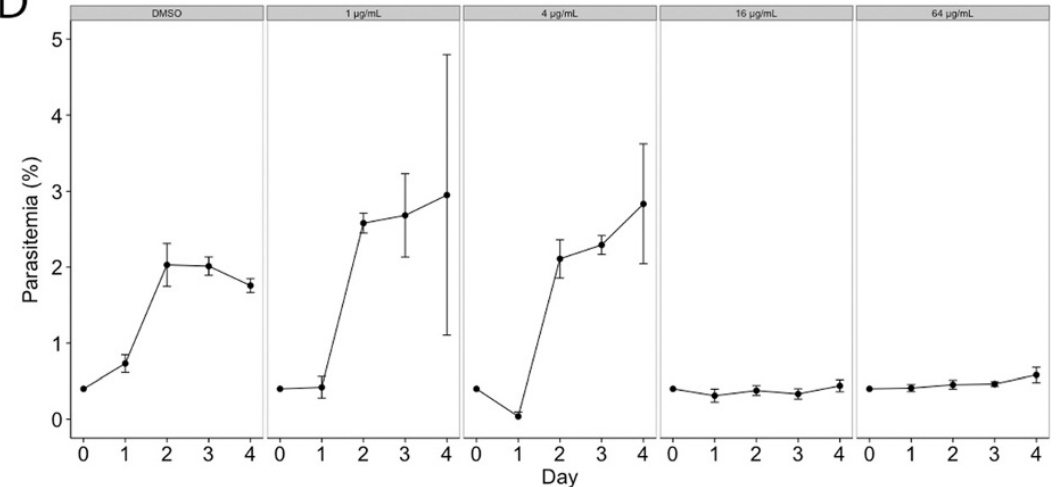

Figure $\mathbf{2}$ (See legend on next page.) 
(See figure on previous page.)

Figure 2 Inhibition of VEGFR-2 but not VEGFR-1 or VEGF reduces parasitaemia in cultures of Plasmodium falciparum. Unspecific inhibition of tyrosine kinases also reduces parasitaemia in cultures. Growth curves of cultures of human blood cells infected with $P$. falciparum, in the presence of bevacizumab (A), SU5416 (B), anti-VEGFR-1 (C), or genistein (D) in increasing concentrations. Controls contain only PBS or DMSO respectively. Inhibition of VEGF by bevacizumab (A) did not affect parasitaemia, whereas inhibition of VEGFR-2 by SU5416 (B) resulted in a significantly reduced parasitaemia in a dose-dependent manner compared to DMSO controls. Inhibition of VEGFR-1 by anti-VEGFR-1 (C) did not replicate this effect and had no effect on parasitaemia. Addition of the unspecific tyrosine kinase inhibitor genistein (D) resulted in a significant reduction in parasitaemia compared to DMSO.

\section{Statistics}

Data did not meet the criteria for using parametric tests. Kruskal-Wallis test was applied for comparing multiple groups and Mann-Whitney $\mathrm{U}$ test was used post hoc. A p-value less than 0.05 was considered statistically significant. All statistical analyses were performed with the R-commander software [18].

\section{Results}

\section{VEGF uptake in PRBCs}

Culture of $P$. falciparum in serum-enriched growth medium resulted in uptake of VEGF into PRBCs, determined by immunocytochemistry on day 6 after the first addition of serum (Figure 1). Neither uninfected red blood cells nor free parasites stained positive for VEGF (Figure 1). This was confirmed by flow cytometry. Confocal microscopy at high magnification showed that VEGF was located intracellularly, in relation to the parasites (Additional file 1: Movie 1). Pre-incubation of the primary anti-VEGF antibody with an excess of murine VEGF prior to incubation almost completely abolished staining for VEGF. The incidence of VEGF uptake was significantly increased in late stages of the malaria parasite (with detection of VEGF in $60.7 \%$ of PRBCs), compared to early stages (VEGF in $6.7 \%$ of PRBCs, $\mathrm{p}=0.038$, Figure $1 \mathrm{E}$ ).

\section{Direct inhibition of VEGF by bevacizumab does not reduce parasitaemia}

Direct inhibition of VEGF in the growth medium was obtained by adding the humanized monoclonal antiVEGF antibody bevacizumab. Flow cytometric analyses showed that parasitaemia did not change significantly compared to PBS-treated controls on any day until the experiment was ended ( $\mathrm{p}>0.06$, Figure $2 \mathrm{~A}$ ).

\section{Inhibition of VEGFR-2 by SU5416 significantly reduces parasitaemia}

Inhibition of VEGFR-2 was obtained by addition of the VEGFR-2 inhibitor SU5416 to the growth medium. This treatment resulted in a significant and dose dependent decrease in parasitaemia as compared to DMSO treated controls from day 2 and onwards ( $<<0.05$, Figure $2 \mathrm{~B}$ ). At day 2 all doses but $4 \mu \mathrm{M}$ resulted in significant growth retardation $(\mathrm{p}<0.01)$. On day 3 and 4 only the two highest doses (100 and $500 \mu \mathrm{M})$ significantly reduced growth $(\mathrm{p}<0.01)$, while at $20 \mu \mathrm{M}$ it was statistically indistinguishable from DMSO $(\mathrm{p}>0.12)$. Four $\mu$ M SU5416 did not reduce the parasitaemia in culture compared to control during experiments ( $p>0.07$, Figure $2 B$ ).

\section{Direct inhibition of VEGFR-1 does not reduce parasitaemia}

Direct inhibition of VEGFR-1 in the growth medium was obtained by adding a peptide binding to VEGFR-1 thereby inhibiting its interaction with VEGF. This treatment did not significantly affect parasite growth compared to PBStreated controls on any day of the experiment $(p>0.06$, Figure 2C).

\section{Treatment with bevacizumab, SU5416 or anti-VEGFR-1 does not cause reduced VEGF accumulation}

The intracellular accumulation of VEGF was studied in parallel with the progression of parasitaemia by flow cytometry analyses of the median fluorescence intensity after staining. This did not reveal any reduction in VEGF accumulation after four days of treatment with bevacizumab $(\mathrm{p}>0.51)$, SU5416 ( $\mathrm{p}>0.06$ ), or anti-VEGFR-1 ( $>$ > 0.34) compared to DMSO and PBS controls respectively (Figure 3A-C). The flow cytometric results were confirmed by immunocytochemistry, showing VEGF staining of PRBCs despite treatment with SU5416 (Figure 1B) or bevacizumab (Figure 1C).

\section{DNA content of PRBCs upon SU5416 and bevacizumab stimulation}

Previous studies have indicated that VEGF is trophic for parasite growth [11]. One mechanism by which SU5416 could reduce parasite growth could be through reduction of the number of merozoites per schizont, which has previously been shown to affect parasite growth rate [19], thus reducing the number of newly infected red blood cells after schizont rupture. However, analyses of flow cytometric data (median fluorescence intensity of DNA binding dye Syto-9) did not support that either SU5416 $(\mathrm{p}=0.054)$ or bevacizumab $(\mathrm{p}=0.095)$ reduced the DNA content of the PRBCs.

\section{Genistein dose-dependently decreases parasitaemia}

Since no association between inhibition of parasite growth and intracellular VEGF was found, an alternative antiparasitic mechanism of SU5416 was sought. SU5416 inhibits VEGFR-2 signalling as a tyrosine kinase inhibitor. 


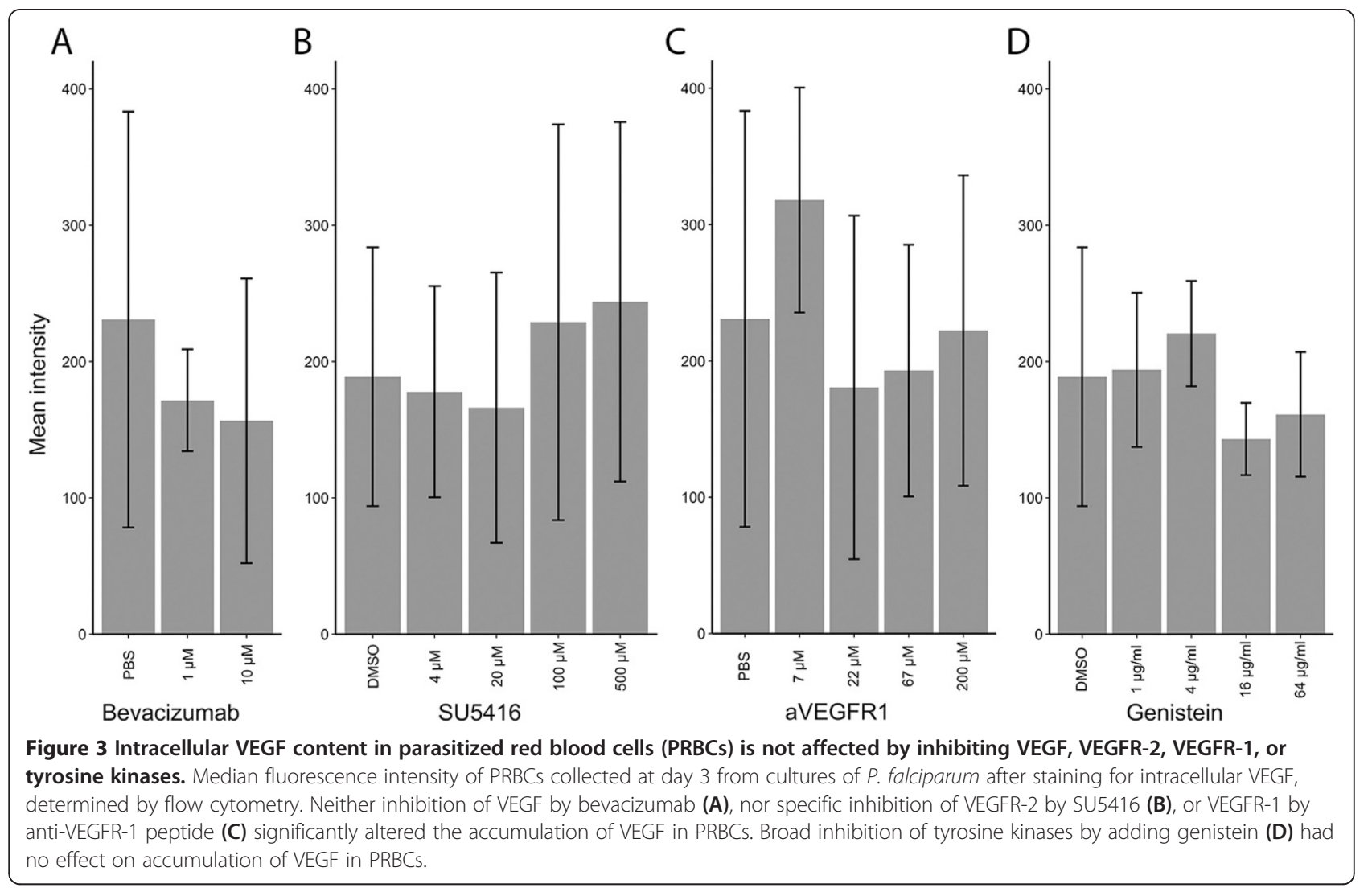

Therefore, the effect of genistein, a broad-acting tyrosine kinase inhibitor, on intra-erythrocytic growth of $P$. falciparum was tested. As seen in Figure 2D genistein inhibited growth significantly in a dose-dependent manner $(\mathrm{p}<0.02)$, while not preventing VEGF accumulation in PRBCs (Figure 3D).

\section{SU5416 and genistein dose-dependently reduce phosphorylation of tyrosine}

The effect of SU5416 on tyrosine phosphorylation was investigated and compared with the other treatments applied. SU5416 and genistein dose-dependently reduced tyrosine phosphorylation in PRBCs (Figure 4A-B), whereas anti-VEGFR-1 peptide and bevacizumab did not show any effect on this parameter (Figure 4C-D).

\section{Uptake of VEGF into red blood cells infected with Plasmodium berghei ANKA}

The murine malaria parasite $P$. berghei ANKA, when infecting mice in vivo, internalized VEGF similarly to the in vitro experiments. Peripheral blood smears, collected after eight days of infection, showed a marked uptake of VEGF into PRBCs, primarily in schizonts (Figure 1D). Confocal microscopy showed that VEGF was located intracellularly (Additional file 2: Movie 2). Analogous to the in vitro results with $P$. falciparum, non-PRBCs and free parasites did not stain for VEGF (Figure 1D).

\section{Discussion}

The presented results strengthen the evidence of VEGF uptake during in vitro culture of P. falciparum [11] and suggest that uptake of VEGF depends on parasite maturity. However, the mechanism behind this uptake remains unclear. This may not be an active process since applying an excess level of anti-VEGF antibody neither affects growth nor intracellular VEGF levels. A dedicated VEGF transporter would likely be hindered by VEGF being of different size or having potential binding sites blocked by the antibody. It is known that the red blood cell membrane in late-stage parasites becomes leaky [20], which could augment internalization of VEGF. Similarly, there is an intracellular accumulation of VEGFR-1 in late-stage parasites (unpublished observation). Previous reports suggest that VEGF acts as a trophic factor for parasite growth since addition of VEGF to an in vitro parasite culture increased parasitaemia and partly rescued the culture from pre-treatment with artesunate [11]. As it was not possible to reduce intracellular VEGF, this effect could not be assessed further.

Besides being a trophic factor, a possible advantage of VEGF accumulation could be the removal of VEGF from 


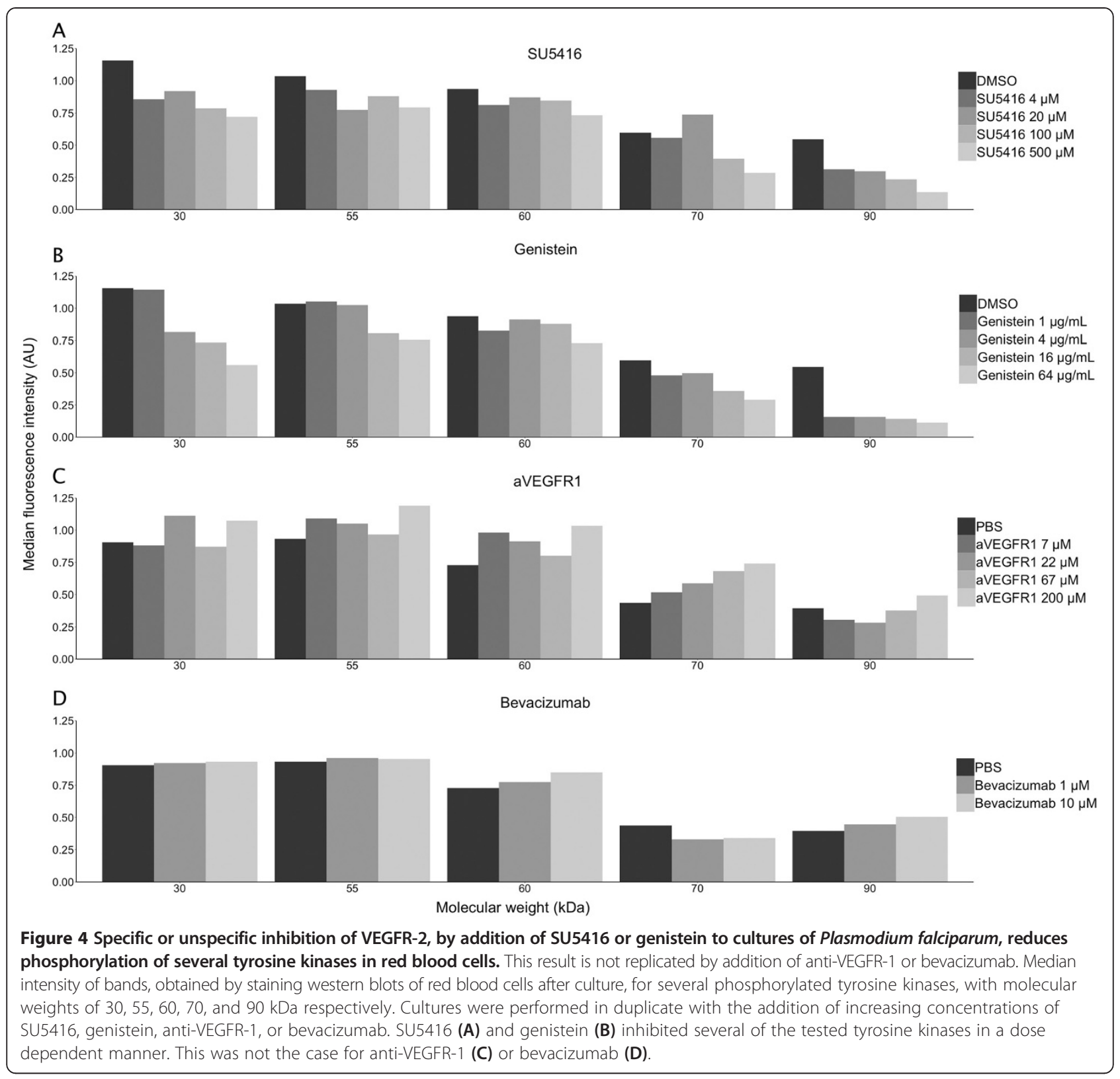

the micro-environment in small brain vessels. Neuronal and epithelial VEGF production is increased during cerebral hypoxia, resulting in nitric oxide (NO)-dependent vasodilation, increased angiogenesis, and thus increased oxygen delivery to the tissue [6]. Because of the microaerophilic nature of $P$. falciparum [21], these changes are potentially detrimental to the parasite, making it a good survival strategy to remove VEGF.

Avoidance of the host immune system is a third possible rationale for VEGF uptake. VEGF is known to stimulate monocyte and T-cell chemotaxis [22,23]. Circulating monocytes and resident macrophages contribute to parasite clearance by phagocytizing PRBCs, and are present in increased numbers in cerebral vessels with sequestration [24]. Thus, removal of VEGF from the cerebral micro-environment could reduce local host immune responses and allow parasite sequestration in the cerebral microvasculature.

Confocal microscopy proved that VEGF was located intracellularly in the PRBCs. This excludes that positive staining for VEGF in PRBCs was due to adsorption onto the PRBC surface rather than absorption. Confocal microscopy did not allow to determine the precise intracellular distribution of VEGF. However it did show that at least some VEGF is present in the parasitophorous vacuoles.

Both microscopy and flow cytometry showed that VEGF uptake was not significantly reduced despite 
VEGF immobilization by a monoclonal antibody, and neither by VEGFR-1 nor VEGFR-2 inhibition. However, inhibition of VEGFR-2 signalling significantly decreased parasitaemia in this study. The antiparasitic action of VEGFR-2 inhibition despite unchanged VEGF accumulation suggests that this effect is not directly related to uptake of VEGF. Instead, SU5416 possibly inhibits the intracellular downstream signalling cascade relating to VEGFR-2 activation. VEGFR-2 is a receptor tyrosine kinase. It exerts its effects, e.g. angiogenesis and tumour growth, via intracellular phosphorylation of tyrosine $[25,26]$. Indeed, P. falciparum also relies on tyrosine kinase activity [27]. Co-culturing P. falciparum with genistein, a broad-acting tyrosine kinase inhibitor, dosedependently reduced parasite growth, as previously shown $[28,29]$. Western blotting of phosphotyrosine levels in late-stage parasites supported the concept that SU5416 directly inhibited parasite tyrosine kinases, as several loci had reduced phosphotyrosine levels. Comparable results were obtained with genistein though it seemed to inhibit a broader range of kinases. Additional research is needed to further assess the association between tyrosine kinase inhibition and the anti-parasitic effect of SU5416 and genistein.

Many studies on the pathogenesis of $\mathrm{CM}$ are performed in a mouse model, infected with the rodent Plasmodium strain $P$. berghei ANKA, because of its accessibility as well as clinical and histopathological similarities to human CM [30]. The presented results show in vivo uptake of VEGF into murine PRBCs infected with $P$. berghei ANKA, similar to human PRBCs. VEGF was located intracellularly in late-stage PRBCs. Thus future studies of VEGF inhibitors may be performed in vivo using this experimental model.

In summary, inhibition of VEGFR-2 signalling reduces intra-erythrocytic growth of $P$. falciparum, but this reduction does not correlate with intracellular levels of VEGF. Rather, it may depend on inhibition of parasite tyrosine kinase activity. The effect of VEGF on parasite growth remains to be clarified. Since VEGF also accumulates in late-stage $P$. berghei ANKA PRBCs in vivo, the relevance of VEGF signalling may be addressed in experimental infections.

\section{Additional files}

Additional file 1: Movie 1. Z-stack obtained by confocal microscopy showing intracellular VEGF in cultured human red blood cells infected with Plasmodium falciparum. DNA is stained blue and VEGF is stained green by immunocytochemistry. The stack shows VEGF located intracellularly. The z-stack was generated with Nikon EZ-C1 software with z-steps of $0.15 \mu \mathrm{m}$.

Additional file 2: Movie 2. Z-stack obtained by confocal microscopy showing intracellular VEGF in murine red blood cells infected with Plasmodium berghei ANKA during experimental CM. DNA is stained blue and VEGF is stained green by immunocytochemistry. The stack confirms that VEGF is located intracellularly and shows a similar pattern of distribution as cultured human red blood cells infected with $P$. falciparum. The z-stack was generated with Nikon EZ-C1 software with z-steps of $0.15 \mu \mathrm{m}$.

\section{Competing interests}

The authors declare that they have no competing interests.

\section{Authors' contributions}

$\mathrm{CH}$ and $\mathrm{NH}$ designed and carried out the experiments, performed the statistical analysis and drafted the manuscript. TS and JK participated in the design, coordination, and interpretation of the study. All authors read and approved the final manuscript.

\section{Acknowledgements}

The use of the Chemidoc XRS at CIM, University of Copenhagen is highly acknowledged.

Received: 11 February 2014 Accepted: 20 May 2014

Published: 28 May 2014

\section{References}

1. Wassmer SC, Moxon CA, Taylor T, Grau GE, Molyneux ME, Craig AG: Vascular endothelial cells cultured from patients with cerebral or uncomplicated malaria exhibit differential reactivity to TNF. Cell Microbiol 2011, 13:198-209.

2. Turner L, Lavstsen T, Berger SS, Wang CW, Petersen JE, Avril M, Brazier AJ, Freeth J, Jespersen JS, Nielsen MA, Magistrado P, Lusingu J, Smith JD, Higgins MK, Theander TG: Severe malaria is associated with parasite binding to endothelial protein C receptor. Nature 2013, 498:502-505.

3. Murphy SC, Breman JG: Gaps in the childhood malaria burden in Africa: cerebral malaria, neurological sequelae, anemia, respiratory distress, hypoglycemia, and complications of pregnancy. Am J Trop Med Hyg 2001, 64:57-67.

4. Deininger MH, Winkler S, Kremsner PG, Meyermann R, Schluesener HJ: Angiogenic proteins in brains of patients who died with cerebral malaria. J Neuroimmunol 2003, 142:101-111.

5. Casals-Pascual C, Idro R, Gicheru N, Gwer S, Kitsao B, Gitau E, Mwakesi R, Roberts DJ, Newton CR: High levels of erythropoietin are associated with protection against neurological sequelae in African children with cerebral malaria. Proc Natl Acad Sci U S A 2008, 105:2634-2639.

6. Ferrara N, Davis-Smyth $\mathrm{T}$ : The biology of vascular endothelial growth factor. In Endocr Rev 1997, 18:4-25.

7. Hellwig-Burgel T, Stiehl DP, Wagner AE, Metzen E, Jelkmann W: Review: hypoxia-inducible factor-1 (HIF-1): a novel transcription factor in immune reactions. J Interferon Cytokine Res 2005, 25:297-310.

8. Yano K, Liaw PC, Mullington JM, Shih SC, Okada H, Bodyak N, Kang PM, Toltl L, Belikoff B, Buras J, Simms BT, Mizgerd JP, Carmeliet P, Karumanchi SA, Aird WC: Vascular endothelial growth factor is an important determinant of sepsis morbidity and mortality. J Exp Med 2006, 203:1447-1458.

9. Medana IM, Day NP, Roberts R, Sachanonta N, Turley H, Pongponratn E, Hien T, White NJ, Turner GD: Induction of the vascular endothelial growth factor pathway in the brain of adults with fatal falciparum malaria is a non-specific response to severe disease. Histopathology 2010, 57:282-294.

10. Warrell DA, White NJ, Veall N, Looareesuwan S, Chanthavanich P, Phillips RE, Karbwang J, Pongpaew P, Krishna S: Cerebral anaerobic glycolysis and reduced cerebral oxygen transport in human cerebral malaria. Lancet 1988, 2:534-538.

11. Sachanonta N, Medana IM, Roberts R, Jones M, Day NP, White NJ, Ferguson DJ, Turner GD, Pongponratn E: Host vascular endothelial growth factor is trophic for Plasmodium falciparum-infected red blood cells. Asian Pac J Allergy Immunol 2008, 26:37-45.

12. Trager W, Jenson JB: Cultivation of malarial parasites. Nature 1978, 273:621-622.

13. Lambros C, Vanderberg JP: Synchronization of Plasmodium falciparum erythrocytic stages in culture. J Parasitol 1979, 65:418-420.

14. Bae DG, Kim TD, Li G, Yoon WH, Chae CB: Anti-flt1 peptide, a vascular endothelial growth factor receptor 1-specific hexapeptide, inhibits tumor growth and metastasis. Clin Cancer Res 2005, 11:2651-2661.

15. Schneider CA, Rasband WS, Eliceiri KW: NIH Image to ImageJ: 25 years of image analysis. Nat Methods 2012, 9:671-675. 
16. Hein-Kristensen L, Wiese L, Kurtzhals JA, Staalsoe T: In-depth validation of acridine orange staining for flow cytometric parasite and reticulocyte enumeration in an experimental model using Plasmodium berghei. Exp Parasitol 2009, 123:152-157.

17. Schindelin J, Arganda-Carreras I, Frise E, Kaynig V, Longair M, Pietzsch T, Preibisch S, Rueden C, Saalfeld S, Schmid B, Tinevez JY, White DJ, Hartenstein V, Eliceiri K, Tomancak P, Cardona A: Fiji: an open-source platform for biological-image analysis. Nat Methods 2012, 9:676-682.

18. R Core Team: R: A language and environment for statistical computing. Vienna, Austria; 2014. [http://www.R-project.org]

19. Reilly HB, Wang H, Steuter JA, Marx AM, Ferdig MT: Quantitative dissection of clone-specific growth rates in cultured malaria parasites. Int J Parasitol 2007, 37:1599-1607.

20. Kutner S, Baruch D, Ginsburg H, Cabantchik Zl: Alterations in membrane permeability of malaria-infected human erythrocytes are related to the growth stage of the parasite. Biochim Biophys Acta 1982, 687:113-117.

21. Scheibel LW, Ashton SH, Trager W: Plasmodium falciparum: microaerophilic requirements in human red blood cells. Exp Parasitol 1979, 47:410-418.

22. Clauss M, Gerlach M, Gerlach H, Brett J, Wang F, Familletti PC, Pan YC, Olander JV, Connolly DT, Stern D: Vascular permeability factor: a tumor-derived polypeptide that induces endothelial cell and monocyte procoagulant activity, and promotes monocyte migration. J Exp Med 1990, 172:1535-1545.

23. Barleon B, Hauser S, Schollmann C, Weindel K, Marme D, Yayon A, Weich HA: Differential expression of the two VEGF receptors flt and KDR in placenta and vascular endothelial cells. J Cell Biochem 1994, 54:56-66.

24. Pongponratn E, Turner GD, Day NP, Phu NH, Simpson JA, Stepniewska K, Mai NT, Viriyavejakul P, Looareesuwan S, Hien TT, Ferguson DJ, White NJ: An ultrastructural study of the brain in fatal Plasmodium falciparum malaria. Am J Trop Med Hyg 2003, 69:345-359.

25. Fong TA, Shawver LK, Sun L, Tang C, App H, Powell TJ, Kim YH, Schreck R, Wang X, Risau W, Ullrich A, Hirth KP, McMahon G: SU5416 is a potent and selective inhibitor of the vascular endothelial growth factor receptor (Flk-1/KDR) that inhibits tyrosine kinase catalysis, tumor vascularization, and growth of multiple tumor types. Cancer Res 1999, 59:99-106.

26. Mendel DB, Laird AD, Smolich BD, Blake RA, Liang C, Hannah AL, Shaheen RM, Ellis LM, Weitman S, Shawver LK, Cherrington JM: Development of SU5416, a selective small molecule inhibitor of VEGF receptor tyrosine kinase activity, as an anti-angiogenesis agent. Anticancer Drug Res 2000, 15:29-41.

27. Solyakov L, Halbert J, Alam MM, Semblat JP, Dorin-Semblat $D$, Reininger $L$, Bottrill AR, Mistry S, Abdi A, Fennell C, Holland Z, Demarta C, Bouza Y, Sicard A, Nivez MP, Eschenlauer S, Lama T, Thomas DC, Sharma P, Agarwal S, Kern S, Pradel G, Graciotti M, Tobin AB, Doerig C: Global kinomic and phosphoproteomic analyses of the human malaria parasite Plasmodium falciparum. Nat Commun 2011, 2:565.

28. Dluzewski AR, Garcia CR: Inhibition of invasion and intraerythrocytic development of Plasmodium falciparum by kinase inhibitors. Experientia 1996, 52:621-623.

29. Kraft C, Jenett-Siems K, Siems K, Gupta MP, Bienzle U, Eich E: Antiplasmodial activity of isoflavones from Andira inermis.J Ethnopharmacol 2000, 73:131-135.

30. Hunt NH, Grau GE, Engwerda C, Barnum SR, der HH V, Hansen DS, Schofield L, Golenser J: Murine cerebral malaria: the whole story. Trends Parasitol 2010, 26:272-274.

doi:10.1186/1475-2875-13-201

Cite this article as: Hempel et al.: Effects of the vascular endothelial growth factor receptor-2 (VEGFR-2) inhibitor SU5416 on in vitro cultures of Plasmodium falciparum. Malaria Journal 2014 13:201. 